(Aus dem physiologischen Institut der Universität Tübingen.)

\title{
Über \\ eine einfache quantitative Trypsinbestimmung und das Fermentgesetz des Trypsins.
}

Von

Dr. Alexander Palladin aus Petersburg.

Einleitung.

Gleichwie das Pepsin in saurer Lösung die Eiweisskörper auflöst und schliesslich in die sogenannten Peptone überführt, so greift das von der Bauchspeicheldrüse gelieferte Ferment, das Trypsin, die Eiweisskörper in neutraler oder schwach alkalischer Lösung in ähnlicher Weise an, löst sie also vor allen Dingen auf, wandelt sie zunächst ebenfalls in peptonähnliche Körper um wie das Pepsin, bleibt aber bei dieser Zersetzung nicht stehen, sondern zertrümmert sie gewissermaassen noch weiter, indem es scbliesslich Aminosäuren und ähnliche verhältnismässig einfache Stoffe aus ihnen bildet.

Will man tryptische Wirkungen im allgemeinen feststellen, so begnügt man sich meistens mit dem Nachweis, dass die Eiweisskörper in schwach alkalischer Flüssigkeit aufgelöst werden, wobei die Lösung in anderer Weise stattfindet als in pepsinhaltigen Flüssigkeiten. Das Trypsin zertrümmert mehr, wie das ja auch der von Kühne geschaffene Name besagen soll, die Eiweisskörper, namentlich das Fibrin, in kleine Krümelchen; das Pepsin löst sie von den Rändern her allmählich auf. Beide Fermente aber lösen unter sonst gleichen Bedingungen in gleichen Zeiten um so mehr Eiweiss auf, je grösser - bis zu einem gewissen Grade - die Fermentmengen sind.

Für das Pepsin glaubt man da eine bestimmte Regel gefunden zu haben, indem Schütz und später Borissow zeigen konnten, dass die von bestimmten Pepsinkonzentrationen gelösten Eiweissmengen nicht wie diese selbst, sondern viel langsamer, nämlich nur 
wie ihre Quadratwurzeln zunahmen, die 1, 4, 9, 16 fach so starke Pepsinlösung also nur die 1, 2, 3, 4fache Eiweissmenge löste. Dieses Gesetz ist aber, wie Grützner ${ }^{1}$ ) zeigte, nur innerhalb gewisser Grenzen ungefä $h r$ richtig, wie auch aus allen von den verschiedensten Forschern angestellten Versuchen ${ }^{2}$ ) hervorgeht, sobald man dieselben genau nachrechnet. Namentlich verdauen die starken Pepsinlösungen durchweg verhältnismässig $\mathrm{zu}$ wenig ${ }^{3}$ ). Innerhalb einer gewissen Grösse der Pepsinmengen stimmt die Regel u u ge fä $\mathrm{hr}$ deshalb, weil die Peptone die Wirkung des Pepsins hemmend beeinflussen. Beseitigt man diese schädigende Wirkung, so sind, wie Grützn er gezeigt hat, die in der Zeiteinheit gelösten Eiweissmengen den wirksamen Pepsinmengen direkt proportional. Wirksame Pepsinmengen sind aber, wenn es sich um Verdauung von festem Eiweiss handelt, natürlich nicht gleichbedeutend mit Pepsinkonzentrationen. Entgegen diesen Auffassungen, dass unter allen Umständen die verdanten Eiweissmengen hinter den Pepsinkonzentrationen bedeutend zurückbleiben, ist zwar neuerdings von $G \operatorname{ross}^{4}$ ) ganz allgemein behauptet worden, dies sei nicht der Fall, sondern "die Pepsinverdauung gehe nach einfachen Proportionen vor sich", wie

1) P. v. Grützner, Versuche und Betrachtungen über meine Methode, Pepsin kalorimetrisch zu bestimmen. Arch. d. Fisiologia vol. 7 p. 223.1909.

2) Vgl. z. B. die Arbeiten von Borissow, Korn, Volhard u. a., die in Grützner's obiger Arbeit besprochen sind.

3) So verhielten sich z. B. in den Samojloff'schen Versuchen (Archives des scienc. biolog. t. 2 p. 727. 1893) die Pepsinmengen wie:

$1: 2: 4: 8: 16: 32$,

die verdauten reduzierten Eiweissmengen in mm wie: $1: 1,19: 1,81: 2,48: 3,13: 3,92$. Nach dem Quadratwurzelgesetz sollen sie sich ver-

halten wie:

$1: 1,4: 2,0: 2,8: 4,0: 5,6$

oder in einem anderen Versuch die Ferment-

mengen wie: $\quad 1: 2: 4: 8: 16: 32: 64$;

die verdauten Fiweissmengen wie: $\quad 1,0: 1,28: 1,72: 2,25: 2,93: 3,73: 4,87$;

die geforderten Eiweissmengen sind: $1,0: 1,4: 2,0: 2,8: 4,0: 5,6: 8,0$,

also ungeheures Zurückbleiben in der Tätigkeit der starken Pepsinlösungen. Oder in einem dritten Versuch mit geringeren Pepsinmengen, die Fermentmengen wie:

$1: 2: 4: 8: 16: 32: 64: 128$

die verdauten Eiweissmengen wie: $1: 1,39: 1,95: 2,79: 3,92: 5,27: 6,56: 8,62$; die geforderten Fiweissmengen wie: $1: 1,4: 2,0: 2,8: 4,0: 5,6: 8,0: 11,24$; hier nur ein geringes Zurückbleiben der starken Fermentlösungen.

4) O. Gross, Die Wirksamkeit des Pepsins und eine einfache Methode zu ibrer Bestimmung. Berliner klin. Wochenschr. 1908 S. 643. 
dies auch beim Trypsin stattfinde. Die zwei-, dreifach so starke Pepsinlösung verdaut in gleicher Zeit die zwei- bzw. dreifache Eiweissmenge u. s. f.

Da mich diese Gesetzlichkeiten interessierten und mir eine einfache und genaue Methode, die wirksamen Trypsinmengen zu bestimmen, ähnlich etwa der Grützner'schen kolorimetrischen Pepsinbestimmungsmethode, nicht bekannt war, folgte ich gern einer Aufforderung von Herrn Prof. v. Grä tzner, eine ähnliche Methode auch für das Trypsin auszuarbeiten und die Gesetzlichkeiten bei der Trypsinverdaunng zu studieren. Im Sommer 1910 unternahm ich diese Untersuchungen im physiologischen Institut in Tübingen und hatte mich dabei des dauernden Rates und der Unterstützung von Herrn Prof. v. Grützner zu erfreuen, dem ich auch die Abfassung der nachstebenden kleinen Arbeit verdanke. Ich spreche ihm an dieser Stelle meinen herzlichen Dank für alle seine Bemühungen aus.

\section{1. Über quantitative Trypsinbestimmungen im allgemeinen.}

R. Heidenhain ${ }^{1}$ ) war wohl der erste, welcher die verschieden starken Trypsinwirkungen der Extrakte von Bauchspeicheldrüsen untersuchte. Die Drüsen entstammten Hunden, welche in verschiedenen Verdauungszuständen getötet worden waren, und wurden gewöhnlich nach 24 stündigem Liegen mit der zehnfachen Gewichtsmenge von Glycerin ausgezogen, hierauf die Massen filtriert und die gewonnenen Extrakte in 1,2\% iger Sodalösung mit Fibrin auf ihre Verdauungsfähigkeit geprüft. Dabei stellte sich im allgemeinen heraus, dass die Extrakte der hungernden Hunde stark, die der gefütterten schwach verdauten. In den ersten befand sich demnach viel, in den zweiten wenig Ferment. Genauere Bestimmungen über die relativen Fermentmengen stellte $\mathrm{Heidenhain} \mathrm{nicht} \mathrm{an.}$

Erst später wurde eine grosse Menge dahingehender Versuche von verschiedenen Forschern ausgeführt, die hier erwähnt werden müssen. Da das Trypsin wohl immer den Faserstoff langsamer auflöst als das Pepsin und demzufolge kleinere Trypsinmengen viel schwieriger nachzuweisen sind als ähnliche Pepsinmengen, um so mehr, als sich in den alkalischen Flüssigkeiten Bakterien leicht störend einmischen, war für Grützner, als er kleine Mengen von

1) R. Heidenhain, Beiträge zur Kenntnis des Pankreas. Pflüger's Arch. Bd. 10 S. 557. 1875. 
Trypsin z. B. im Harn nachweisen wollte, eine derartige empfindliche Methode von grossem Wert. Sie wurde im folgenden gefunden und von Gehrig ${ }^{1}$ ), der im hiesigen Institut arbeitete, in seinen Untersuchungen erprobt. Gehrig färbte sauber gewaschenes und fein zerkleinertes Fibrin in alkoholischer Lösung von Magdalarot, einem Farbstoff, der weder in Wasser noch in wässeriger Sodalösung sich auflöst. Wird dieses rot gefärbte Fibrin in Sodalösung eingelegt, wodurch es ein wenig aufquillt, und dann in alkalische Trypsinlösung gebracht, so färbt sich die Flüssigkeit schon bei der Lösung von ganz geringen Fibrinmengen schwach, aber deutlich rot, bei Lösung grösserer Fibrinmengen natürlich mehr oder weniger dunkelrot, wie ganz ähnliches bei der Grützner'schen kolorimetrischen Pepsinmethode stattfindet. Würde sich eine viel grössere ungefärbte Fibrinmenge auflösen, so könnte man davon natürlich nichts sehen, gerade so wenig wie man die Auflösung eines Körnchens Kochsalz im Wasser ohne weiteres sieht, wohl aber die Lösung einer Spur von Methylviolett oder eines anderen ähnlichen Farbstoffes geradezu in die Augen springt ${ }^{2}$ ).

Wie mir aber Herr Prof. v. Grützner mitteilte, hat er das frühere Magdalarot nicht mehr erhalten. Fast alle ebenso benannten Farbstoffe lösten sich leicht in Wasser, waren also ganz unbrauchbar; nur eine einzige Probe von den vielen untersuchten löste sich, allerdings in der ersten Zeit nicht in Wasser, wohl aber dann, wenn man die Mischung schüttelte oder längere Zeit mit Sauerstoff in Berührung brachte. Auch dieser Farbstoff war also unbrauchbar. Nach vielfachen vergeblichen Versuchen meinerseits mit allen möglichen Farbstoffen verschaffte uns Herr Prof. Bülow hierselbst in liebenswürdiger Weise einen Farbstoff, der sich vortrefflich für unseren Zweck eignete und zudem den Vorteil hatte, billig zu sein,

1) F. Gehrig, Über Fermente im Harn. Pflüger's Arch. Bd. 38 S. 35.1886.

2) C. Oppenheimer beschreibt die Methode von Gehrig in seinem Buche (Die Fermente S. 124. Leipzig 1903) folgendermaassen. Er sagt: „Man bringt das gefärbte Fibrin auf ein Filter und beobachtet (zählt eventuell) die gefärbten Tropfen, die bei der Trypsinwirkung aus dem Trichter herauskommen." Davon ist gar keine Rede. Oppenheimer macht hier denselben kaum begreiflichen Fehler, den er bei der Beschreibung meiner kolorimetrischen Pepsinbestimmungsmethode an gleichem Orte macht, bei der ebensowenig filtriert oder getropft wird. (Vgl. meine obengenannte Arbeit im Archivio di fisiologia p. 251.) 
während brauchbares Magdalarot ziemlich teuer war. Dieser Farbstoff ist Spritblau, bezogen von den Farbfabriken aus Elberfeld, wo es in zwei Arten, Spritblau bläulich und Spritblau rötlich, verkauft wird. Beide Arten sind wohl ziemlich gleich gut verwendbar. Ich stellte meine Versuche mit dem Spritblau bläulich an. Es ist, wie notwendig, völlig unlöslich in Wasser, dagegen sehr leicht löslich in Alkohol, ziemlich leicht löslich in Glycerin. In Alkobol gelöst hat es eine schöne violettblaue Farbe, die in starker Verdünnung mit sodahaltigem Wasser versetzt rötlich wird. Diese rötliche Farbe tritt aber erst ein bei Anwendung von 0,5-1,0\% iger Sodalösung. Die von mir angewendete $0,1 \%$ ige Sodalösung verändert seine Farbe nicht.

Ehe ich jedoch zur genaueren Beschreibung dieser Methode übergehe, wird es sich empfehlen, die anderen Methoden kurz zu schildern, deren man sich zum quantitativen Nachweis dieses Fermentes bedient hat.

a) Altere quantitative Methoden zur Bestimmung des Trypsins.

Ich zähle diese Methoden kurz der Zeit nach auf. Fermi ${ }^{\mathbf{l}}$ ) verwendete geronnenen Leim zur Verdauung, der sich in Reagenzgläsern befand. $7 \mathrm{~g}$ reine Gelatine in $100 \mathrm{~g}$ gesättigter wässeriger Thymollösung werden durch Erwärmen verflüssigt, die Lösungen in die Reagenzgläschen gefüllt und, nachdem die Höhe der Gelatine an dem Gläschen bezeichnet war, mit den versehiedenen verdauenden Lösungen (namentlich von Mikroorganismen stammend) übergossen. Der erstarrte Leim verflüssigt sich in Zimmertemperatur durch die Berührung mit der Fermentlösung und zwar um so schneller, je stärker dieselbe ist. Dabei sei bemerkt, dass alle diese Versuche tage-, ja wochenlang dauerten.

Ebenfalls Leim benutzten Hankin und Wesbrook ${ }^{2}$ ), welche eine Glasplatte mit einer dünnen Schicht Thymolgelatine überzogen und Tröpfchen von Fermentlösungen auf die geneigte Platte brachten. „Ein Wassertröpfchen bleibt bei geringer Neigung der Platte an seinem Ort, während ein trypsinhaltiges Tröpfchen sich nach unten

1) Cla udio Fermi, Die leim- und fibrinlösenden usw. Fermente der Mikroorganismen. Arch. f. Hygiene Bd. 10 S. 1. 1890.

2) Hankin et Wesbrook, Annales de l'Institut Pasteur t. 6. 1892. 
zieht." Ich folge der Schilderung von 0 p penheimer, da mir die Originalarbeit nicht zugänglich war.

Auch die Mett'sche Methode, welche zur quantitativen Pepsinbestimmung vielfach, in dem $\mathrm{Pawlow}$ 'schen Laboratorium wohl einzig angewendet worden ist und noch wird, wurde in entsprechender Form verändert, auch für das Trypsin benutzt. Die Kapillarröhrchen, welche in bekannter Art mit geronnenem Hühnereiweiss gefullt waren, legte man in schwach alkalische Trypsinlösungen und liess ihren Inhalt bei Körpertemperatur verdauen. S a mojl of $\mathrm{f}^{1}$ ) empfabl zuerst diese Methode für das Trypsin, ohne irgendwie nähere Angaben zu machen. Späterhin hat $\mathrm{W}$ alther ${ }^{2}$ ) die Methode weiter angewendet, indem er die Mett'schen Röhrchen in verschieden verdünnte Pankreassäfte legte und die Längen der abverdauten Eiweisszylinder maass. Er fand, was für uns von besonderer Wichtigkeit ist, die Schütz-Borrissow'sche Regel auch hier bestätigt. Die verdauten Eiweissmengen verhielten sich wie die Quadratwurzeln aus den Fermentmengen bzw. deren Konzentrationen.

Linossier ${ }^{3}$ ) ersetzte das durch die Hitze geronnene Eiweiss durch erstarrten Leim, welcher mit Methylviolett gefärbt war. Er wird flüssig in die Gläschen eingesaugt und gerinnt in niederer Temperatur. Weiteres ist mir nicht bekannt.

Weiterhin hat Vernon ${ }^{4}$ ) eine grosse Reihe von eingehenden Versuchen über die Trypsinwirkung angestellt, von denen das Wesentliche und das uns besonders Angehende hier mitgeteilt sei. Vernon benutzte fein gehacktes Fibrin, welches er mit alkalischer Trypsinlösung verdaute und die verdaute sowie die ursprüngliche Menge in hier nicht näher zu beschreibender Weise durch Zentrifugierung feststelite. Er fand in Übereinstimmung mit den Angaben von Heidenhain, dass das Trypsin in Pankreasextrakten in $0,4 \%$ iger Sodalösung bei der Temperatur von $38^{\circ}$ schnell zerstört wird und ferner, dass die verdauten Fibrinmengen der Schütz-Borissow'schen Regel folgen, also nur mit den Quadratwurzeln der Fermentmengen wachsen.

1) Samojloff, Détermination du pouvoir fermentatif des liquides etc. par le procédé de M. Mette. Arch. d. scienc. biolog. t. 2 p. 699.1893.

2) A. A. Walther, Arch. d. scienc. biolog. t. 7 p. 15.1899.

3) Linossier, Compt. rend. de la société de biol. t. 52 p. 298.1900. Zitiert nach 0 ppenheimer.

4) H. M. Vernon, The conditions of action of Trypsin on fibrin. The journ. of Physiol. vol. 26 p. 405. 1900-1901. 
Thomas und Weber ${ }^{1}$ ) verwenden nun zum erstenmal einen neuen Eiweisskörper, nämlich Kasein in alkalischer Flüssigkeit gelöst. Diese Lösungen werden mit grösseren oder kleineren Fermentmengen versetzt und mehr oder weniger verdaut. Nach einer bestimmten Zeit wird das noch unverdaute Kasein ausgefällt. Selbstverständlich ist der ausgefällte Niederschlag um so bedeutender, je geringer die Verdauung war. Der Niederschlag wird auf gewogenen Faltenfiltern gesammelt, mit destilliertem Wasser gewaschen, getrocknet und gewogen, also eine Reihe recht umständlicher Operationen vollzogen.

Löhlein ${ }^{2}$ ) hat auf Anregung von Volhard diese Methode vereinfacht. Er verfährt in der Hauptsache folgendermaassen: Die Verdauung in den alkalischen Flüssigkeiten wird unterbrochen durch Zusatz einer bestimmten Menge Salzsäure und Glaubersalz, welche das unverdaute Kasein ausfällen. „Die salzsauren Peptone gehen durchs Filter, und der durch sie bedingte (durch Titration festzustellende) Säurezuwachs dient als Maass für den Grad der Fermentwirkung."

Der Kaseinniederschlag, welcher die Salzsäure zurückhält, ist um so geringer und das Filtrat um so saurer, je mehr Kasein verdaut wurde.

Löhle in findet, und das interessiert uns hier am meisten, dass die Schütz-Borissow'sche Regel für das Trypsin nicht gilt, sondern dass eine einfache direkte Proportionalität besteht zwischen den Mengen des Fermentes und dem von ihm verdauten Eiweiss.

Zu ganz demselben Frgebnis kommt durch eine grosse Zahl von Versuchen mit verschiedenen Trypsinlösungen, sog, „Ölmagensäften", vermittelst derselben $\mathrm{Volh}$ ard'schen Methode $\mathrm{Faubel}{ }^{3}$ ). Er sagt: "Meine Versuche ergeben, dass das Schütz-Borissow'sche Wurzelgesetz, wie es bei der Pepsin- und Steapsinverdauung zutrifft, bei dem Trypsin seine Gültigkeit nicht findet, dass hier vielmehr die

1) Thomas und Weber, Zentralbl. f. Stoffwechsel- u. Verdauungskrankh. Bd. 2. 1901. Zitiert nach Löhlein.

2) W. Löhlein, Über die Volhard'sche Methode der quantitativen Pepsin- und Trypsinbestimmung durch Titration. F. Hofmeister's Beiträge zur chem. Physiol. usw. Bd. 7 S. 120.1905.

3) O. Faubel, Untersuchungen über den menschlichen Banchspeichel und das Fermentgesetz des Trypsins. F. Hofmeister's Beiträge zur chem. Physiologie usw. Bd. 10 S. 35.1907. 
Verdauung bei gleichen Verdauungszeiten direkt proportional den zugefügten Fermentmengen fortschreitet."

Der Vollständigkeit balber sei noch erwähnt, dass zum Nachweis von kleinen Trypsinmengen Bierry und $\mathrm{Henri}{ }^{1}$ ) sowie $\mathrm{Schumm}$ besondere Methoden angegeben haben. Die ersteren verwenden frische Milch nach dem Zentrifugieren und Filtrieren durch feuchte Papierfilter. Die Milch hellt sich auf und wird in 10-15 Minuten durchscheinend. $\mathrm{Schumm}{ }^{2}$ ) zeigt, dass Trypsin in einer konzentrierten Lösung von Witte-Pepton ziemlich schnell Tyrosin bildet, welches man gelegentlich in schönen Drusen kristallisiert nachweisen kann.

Eine sinnreiche Methode beschreiben auch Henri und Larguier des Bancels ${ }^{3}$ ), welche in kurzer Zeit zu bestimmten Ergebnissen führt und den Verlauf der Trypsinverdauung deutlich erkennen lässt. Sie bedienen sich der Messung des elektrischen Widerstandes, der sich schon kurze Zeit (10 Minuten) nach dem Beginne der Verdauung auffällig ändert.

Die letzte mir bekannte Arbeit über Trypsinverdaunng verdanken wir $\mathrm{Gross}^{4}$ ). Seine Methode ist verhältnismässig einfach und, wie ich glaube, recht genau. Auch er verwendet Kasein (Caseinum purissimum Grü ble r) in $0,1 \%$ iger Sodalösung (1 Kasein : 1000 Lösung). Die Reagenzọläser werden mit je $10 \mathrm{ccm}$ dieser Kaseinlösung und mit den nötigen Trypsinmengen versetzt. Die Verdauung geschieht bei $40^{\circ} \mathrm{C}$. Je nach der Menge des Fermentes ist nun das Kasein früher oder später verdaut, so dass eine kleine Flüssigkeitsprobe aus dem Verdauungsgläschen mit wenig verdünnter Essigsäure $(1: 40)$ versetzt (viel Essigsäure löst den Niederschlag) nur da einen stärkeren oder schwächeren Niederschlag gibt, wo noch mehr oder weniger Kasein ungelöst vorhanden ist. Ist das Kasein völlig verdaut, so tritt kein Niederschlag ein. Gross fand mit dieser Methode, dass die Verdaungszeiten sich

1) Bierry und Henri, Compt. rend. de la société de biol. t. 52.1902. Zitiert nach Oppenheimer.

2) 0. Schumm, Über menschliches Pankreassekret. Zeitschr. f. physiol. Chemie Bd. 36 S. 292. 1902.

3) V. Henri et Larguier des Bancels, Compt. rend. t. 136 p. 1088. 1903.

4) 0. Gross, Die Wirksamkeit des Trypsins und eine einfache Methode zu ihrer Bestimmung. Arch. f. exper. Pathol. usw. Bd. 58 S. 157. 1908. 
genau umgekehrt proportional verhalten wie die Fermentmengen, also das Schütz-Borissow'sche Gesetz für das Trypsin in keiner Weise Geltung hat.

b) Die kolorimetrische Trypsinbestimmungsmethode im besonderen.

Wie schon oben angedeutet, färbte ich das zu verdauende Fibrin wie Grützner und Gehrig es getan haben, mit einem geeigneten Farbstoff und zwar mit Spritblau. Der Farbstoff wurde in Alkohol aufgelöst und das saubere, zerschnittene und in Glycerin aufbewahrte Fibrin nochmals mit einer Schere fein zerschnitten und in dieser gesättigten Farbstofflösung 48 Stunden liegen gelassen. Hierdurch nimmt es eine schöne dunkelviolette Farbe an.

Dieses blaue Fibrin bewahrte ich in Glycerin auf, in welchem Spritblau bis zur Sättigung gelöst war. Solches Fibrin bleibt weicher und wird besser verdaut als dasjenige, welches dauernd in Alkohol liegt.

Vor Anstellung eines Versuches wird dieses Spritblaufibrin sorgfältig mit Wasser von seinem Glycerin befreit und dann auf etwa eine Stunde in $0,1 \%$ jge Sodalösung gelegt, in welcher es ein wenig aufquillt. Zur Verdauung benutzte ich Pankreatin aus der chemischen Fabrik Rhenania in Aachen, welches sehr kräftig wirkte.

Um einen Überblick über die Verdauung dieses bunten Fibrins $\mathrm{zu}$ haben, wurden folgende Versuche angestellt.

Zunächst wurden gleiche Mengen von Fibrin unter möglichst gleichen Bedingungen, also in genau gleich weiten Reagenzgläsern bei derselben Temperatur der Verdauung durch gleiche Mengen Trypsin ausgesetzt. Die Flüssigkeitsmenge betrug in allen Gläschen $15 \mathrm{ccm}$ und war $0,1 \%$ ige Sodalösung, welche sich mir am besten bewährte. Das am Boden befindliche Fibrin war durchweg $1^{1 / 2} \mathrm{~cm}$ hoch. Nach $10-20$ Minuten wurden alle Gläschen schwach bläulich, und die Farben waren nicht von einander zu unterscheiden. Alle 5-10 Minuten kehrte man die Gläschen um, indem man sie mit dem sauberen Daumen verschloss. Die Verdauung ging in allen Gläschen in gleichem Schritt vorwärts, und die Flüssigkeiten wurden immer dunkler, blieben aber untereinander vollkommen gleich.

Weiter wurden verschiedene Fermentmengen angewendet, und sofort änderte sich natürlich der Verlauf des Versuches. Die 
Gläschen, welche viel Ferment enthielten, wurden schneller blau und waren oft schon recht dunkelblau, während die ersten kaum einen blauen Schimmer zeigten; ein Kontrollgläschen mit der Sodalösung ohne Trypsin blieb farblos.

Um nun die Menge des gelösten Faserstoffs festzustellen, legte ich mir wie Grützer eine Farbenskala an, deren Herstellung aber schwieriger war als diejenige mit Karmin. Ich verfuhr dabei folgendermaassen. Je $0,3 \mathrm{~g}$ abgepresstes Spritblaufibrin werden versetzt mit $13,5 \mathrm{ccm}$ Sodalösung von $0,1 \%$ und $1,5 \mathrm{ccm}$ Pankreatinlösung ( $1 \mathrm{~g}$ Pankreatin auf $100 \mathrm{ccm}$ Sodalösung $0,1 \%$ ) und in sechs Gläschen von $13 \mathrm{~mm}$ Durchmesser verteilt. Die Verdauung schreitet wieder wie in obigem Versuche gleich schnell vorwärts. Nach einigen Stunden ist in allen Gläschen das Fibrin verdaut. Die Flüssigkeiten haben alle die gleiche duukelblane Farbe. Die Inhalte aller Gläschen werden zusammengegossen und aus dieser Flüssigkeit eine Skala aus sechs Gliedern angefertigt.

Gläschen 1 enthielt $1 \mathrm{ccm}$ dieser blanen Flüssigkeit $+14 \mathrm{ccm}$ Wasser,

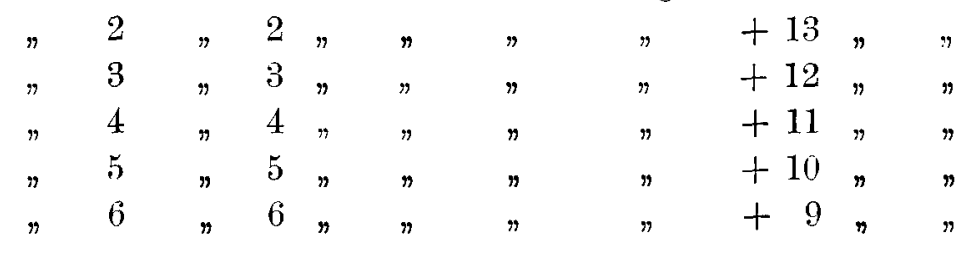

Gläschen 1 enthält demnach gelöst $0,02 \mathrm{~g}$ feuchtes Fibrin und hat Farbe $\mathrm{I}$,

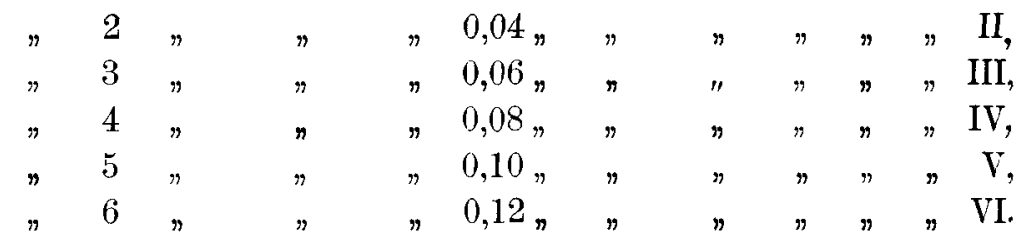

Das Gläschen 1 sieht ganz blassblau-violett aus, ähnlich der Farbe gewisser grossblütiger Campanulablüten (Campanula rapunculoides), die mittleren Gläschen gleichen der Farbe der Rosmarinblüten, und die späteren sind entsprechend dunkler. Über die Haltbarkeit dieser Farblösungen habe ich noch wenig Erfalırung. Sie dürften aber nicht so haltbar sein wie diejenigen der Grützner'schen Karminskala. Meine Zeit war leider zu knapp, um darüber weitere Versuche anzustellen. Jedenfalls hielten sie, im Dunklen aufbewah rt ihre Farben, solange ich sie brauchte. Ich bezeichne die Farben, 
so wie es Grützner getan hat, mit den römischen Zahlen I-VI; I ist die hellste, VI die dunkelste Nüance.

Als ich weitere Versuche anstellte, indem ich in den sechs Gläschen $0,02,0,04$ usw. bis $0,12 \mathrm{~g}$ blaues Fibrin mit je $15 \mathrm{ccm}$ Trypsinlösung verdaute, erhielt ich stets dieselben Farbentöne wie in dem vorigen Versuch. Nur einmal waren die blassblauen Gläser hier eine Spur heller als im ersten Versuch.

Über die Brauchbarkeit der Methode stellte ich nun eine Reihe von Versuchen an, in denen gleiche Fibrin-, aber verschiedene Trypsinmengen Verwendung fanden. Folgender Versuch gibt darüber Aufschluss.

\section{Versuch 1 (21. Mai).}

Gläschen 1 enthält $15 \mathrm{ccm} 0,1 \%$ iger Sodalösung $+0,0 \mathrm{ccm}$ Trypsinlösung von $1 \%$,

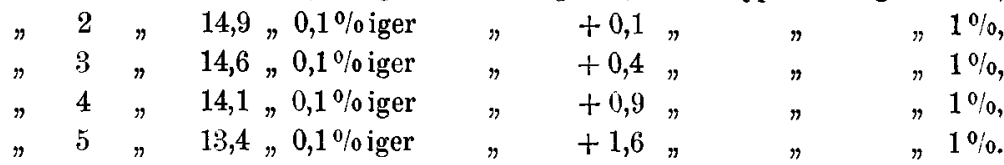

Beginı des Versuches $10^{\mathrm{h}} 20^{\prime}$, die Verdauung geschah hier wie in allen anderen Versuchen bei $40^{\circ} \mathrm{C}$. Um die nachbenannten Zeiten hatten die Gläschen folgende Farben erreicht:

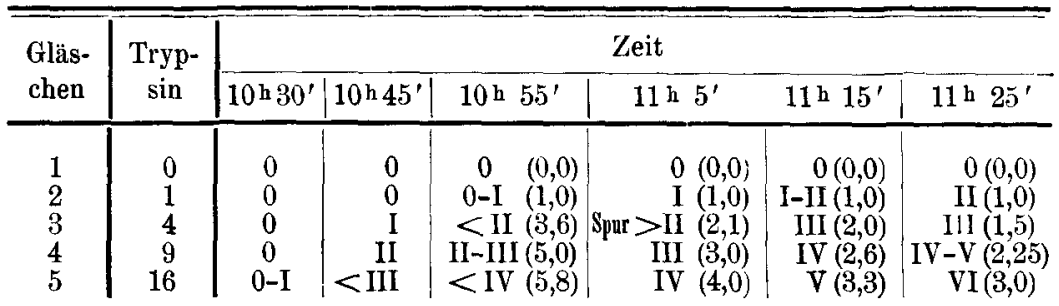

Ein anderer ähnlicher Versuch mit etwas mehr Trypsin bei sonst gleicher Anordnung ist folgender.

Beginn $10^{\text {h }} 43^{\prime}:$

Versuch 2 (6. Juni).

\begin{tabular}{|c|c|c|c|c|c|}
\hline \multirow{2}{*}{ Gläschen } & \multirow{2}{*}{ Trypsin } & \multicolumn{4}{|c|}{ Zeit } \\
\hline & & $10^{\mathrm{h}} 55^{\prime}$ & $11^{\mathrm{h}} 5^{\prime}$ & $11^{\mathrm{b}} 15^{\prime}$ & $11^{\mathrm{h}} 25^{\prime}$ \\
\hline $\begin{array}{l}1 \\
2 \\
3 \\
4 \\
5\end{array}$ & $\begin{array}{r}0 \\
1 \\
4 \\
9 \\
16\end{array}$ & $\begin{array}{c}0 \\
0 \\
0 \\
0 \rightarrow \mathrm{I} \\
\mathrm{I}\end{array}$ & $\begin{array}{c}0 \\
0 \\
0-1 \\
\text { I-III } \\
\text { II-III }\end{array}$ & $\begin{array}{cc}0 & (0,0) \\
\text { L I } & (1,0) \\
\text { I-II } & (1,9) \\
\text { II-III } & (3,1) \\
\text { IV } & (5,0)\end{array}$ & $\begin{array}{cc}0 & (0,0) \\
\text { I-II } & (1,0) \\
\text { III } & (2,0) \\
\text { IV } & (2,6) \\
\text { V-VI } & (3,6)\end{array}$ \\
\hline
\end{tabular}

Farbe 0 bedeutet natürlich farblos, <II bzw. $>$ II bedeutet etwas heller bzw. etwas dunkler als II, I-II eine Farbe etwa in der Mitte zwischen I und II. 
Ein dritter Versuch mit viel weniger Ferment $(0,25 \%)$ ergibt folgende Verbältnisse. Alles übrige ist wie in den beiden vorhergehenden Versuchen.

Beginn 2 h $30^{\prime}$.

Versuch 3 (8. Juni).

\begin{tabular}{|c|c|c|c|c|c|c|c|}
\hline \multirow{2}{*}{ Gläschen } & \multirow{2}{*}{ Trypsin } & \multicolumn{6}{|c|}{ Zeit } \\
\hline & & $2^{\text {h }} 40^{\prime}$ & 2 h $50^{\prime}$ & $3^{h}$ & $\longdiv { 3 \mathrm { h } 1 0 ^ { \prime } }$ & $3^{\text {h }} 20^{\prime}$ & $3^{\text {h }} 30^{\prime}$ \\
\hline 1 & 0 & 0 & 0 & 0 & 0 & $0(0,0)$ & $0(0,0)$ \\
\hline 2 & 0,1 & 0 & 0 & 0 & 0 & $I(1,0)$ & I-II $(1,0)$ \\
\hline 3 & 0,4 & 0 & 0 & 0 & I & J[ $(2,0)$ & III $(2,0)$ \\
\hline 4 & 0,9 & 0 & 0 & I & I-II & $<\mathrm{III}(2,9)$ & $<\operatorname{IV}(2,5)$ \\
\hline 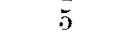 & 1,6 & 0 & $0-\mathrm{I}$ & $<$ III & $<\mathrm{III}$ & $<\operatorname{IV}(3,9)$ & $\mathrm{V}(3,3)$ \\
\hline
\end{tabular}

Was kann man nun aus diesen und einer grossen Anzahl ähnlicher Versuche, welche ich stets mit denselben Ergebnissen angestellt habe, schliessen?

1. Was zunächst die Methode als solche anlangt, so dürfte sie als ausserordentlich einfach zu empfehlen und in dieser Beziehung allen anderen mir bekannten überlegen sein; denn nach kurzer Zeit, nach 10-20 Minuten, weiss man, wo viel, wo wenig, wo gar kein Ferment in der betreffenden Lösung ist, 2. kann man auch den relativen Fermentgehalt verschiedener Flussigkeiten durch Vergleichung mit einer oder mehreren Lösungen von bestimmtem Fermentgehalt unschwer feststellen, 3. ist die Methode auch sehr genau, weil schon ausserordentlich kleine Mengen von Ferment sich durch Blaufärbung der Verdauungsflüssigkeit kenntlich machen.

Von den mir bekannten (oben erwähnten) Methoden kann meines Erachtens nur die Methode von Mett und diejenige von Gross mit der kolorimetrischen in Konkurrenz treten; denn alle anderen Methoden sind sicher nicht genauer, vielfach nicht so genau, aber alle ungemein viel umständlicher als die kolorimetrische Methode. Wenn man schon fällen, filtrieren, trocknen, wägen oder statt der beiden letzteren Operationen auch nur titrieren muss, so ist das namentlich im Vergleich mit dem einfachen Umkehren der Reagenzgläschen und der Feststellung ihres mehr oder weniger gefärbten lnhaltes eine umgeheure Umständlichkeit.

Die Mett'sche Trypsinbestimmungsmethode, namentlich in der von uns später mitzuteileuden Art, ist gut und empfehlenswert, dauert aber so viel Stunden als die kolorimetrische Minuten. 
Die Methode von $G$ ross ist natürlich auch viel umständlicher als die kolorimetrische; denn wenn man, wie das Gross getan hat, für eine einzige Trypsinlösung mehrere (10) Reagenzgläser haben muss, um zu versehiedenen Zeiten die Ausfällung des noch ungelösten Kaseins durch Essigsäure vorzunehmen, so gebraucht man bei fünf verschiedenen Trypsinlösungen und etwa einigen Kontrollgläschen über 50 Reagenzgläser. Auch die Zahl der Fällungen ist nicht unbedeutend und dürfte wohl die Zahl 20 weit übersehreiten, namentlich wenn man es mit unbekannten Fermentmengen zu tun hat, und wenn man, wie es Gross angibt, auf die Sekunde genau den Abschluss des Verdauungsprozesses feststellen will. Das halte ich, wobei ich natürlich auch die Ansichten des Herrn Prof. v. Grützner wiedergebe, überhaupt für unmöglich; denn da die Operationen, die mit der Ausfällung verknüpft sind, mehrere Sekunden in Anspruch nebmen, kann man unmöglich die Beendigung der Verdauung auf eine Sekunde genau angeben.

Es ist aber noch, was nach Grützner für alle quantitativen Fermentbestimmungen mehr oder weniger gilt, darauf hinzuweisen, dass die Methode einen grundsätzlichen Fehler hat, der sich zwar hei ihr in sehr geringem Grade bemerklich macht, aber doch vorhanden ist, das ist der Umstand, dass man die Zeit, in welcher die Verdauung vollkommen abgeschlossen ist oder sein soll, als Maass für die Verdauungskraft verwendet. Es ist dies bei allen Fermentprozessen ausserordentlich schwierig, ja wie ich glaube, kaum möglich. Wer will auch bei der Methode von Gross sagen, ob nicht noch eine Spur unverdautes Kasein in einem Verdauungsgläschen sich befindet, welches durch die Essigsäure nicht nachgewiesen wird und nicht nachgewiesen werden kann? Denn sehr kleine Mengen von ungelöstem Kasein entziehen sich dem Nachweise durch Essigsäure, was leicht zu konstatieren ist.

Nichtsdestoweniger halte ich die Methode von Gross, die man sich übrigens ziemlich vereinfachen kann, für gut und empfehlenswert, aber wie gesagt nicht für genauer und für viel umständlicher als die kolorimetrische. 
c) Das Fermentgesetz des Trypsins.

Bei dem Trypsin, wie bei allen daraufhin untersuchten Fermenten besteht die zuerst von $S_{c h w a n n^{1}}$ ) für das Pepsin festgestellte Tatsache, dass unter sonst gleichen Bedingungen um so mehr bzw. um so schneller die von den Fermenten angegriffenen Stoffe zersetzt werden, je mehr Ferment bis zu einer gewissen Grenze in Wirksamkeit ist. Was für eine Regel oder, wie man vielfach sagt, was für ein Gesetz gilt nun für das Trypsin? Während für das Pepsin alle Forscher mit Ausnahme von Gross darin übereinstimmen, dass die in gleichen Zeiten verdauten Eiweissmengen bedeutend zurückbleiben hinter den Pepsinkonzentrationen und viele das Quadratwurzelgesetz von Schütz-Borissow als genau gültig annehmen, lauten die Angaben der Forscher für das Trypsin so, dass etwa die eine Hälfte derselben auch dieses Gesetz für das Trypsin gelten lassen, wie Pawlow ${ }^{2}$ ), Samojloff ${ }^{3}$ ), Walther ${ }^{3}$ ), Vernon ${ }^{3}$ ), die anderen dagegen, wie $\operatorname{Volhard}^{3}$ ), Löhlein ${ }^{3}$ ), Faubel ${ }^{3}$ ), Gross ${ }^{3}$ ) dieser Annahme ebenso bestimmt widersprechen. Meine nächste Aufgabe, deren Ergebnis Herr Prof. v. Grützner richtig voraussagte, war nun die, diese Widersprüche aufzuklären.

$\mathrm{Zu}$ diesem Behufe machte ich zunächst Versuche mit Trypsin (und nebenbei mit Pepsin) und geronnenem Hühnereiweiss, ähnlich wie es Schwann, Bidder und Schmidt, Ebstein und Grützner getan haben. Das fein zerschnittene, gekochte Hühnereiweiss, immer je $1 \mathrm{~g}$, wurde in Reagenzgläschen in Brutwärme den Verdauungsflüssigkeiteu ausgesetzt und nach einigen Stunden die Menge des nicht verdauten Eiweisses nit der Wage bestimmt. Da es mir nur auf Annäherungswerte ankam, trocknete ich weder das zu verdauende noch das verdaute Eiweiss, um aus dem Trockenrückstand sein Gewicht zu bestimmen, wie es z. B. Ebstein und Grützner getan hatten, sondern wog es einfach feucht $a b$, nachdem es von der Verdauungsflüssigkeit abfiltriert, mit Wasser abgespült und das überflüssige Wasser mit Fliesspapier abgetrocknet war. Bei sauberer und gleichmässiger Handhabung dieser Maassnahmen dürften die Fehler sehr gering sein. Statt vieler Versuche ein paar Beispiele.

1) Th. Schwann, Über das Wesen usw. J. Müller's Arch. f. Anat., Physiol. usw. Jahrg. 1836 S. 90.

2) J. P. Paw low, Die Arbeit der Verdaungsdrüsen S. 33. Wiesbaden 1898.

3) Siehe oben S. 341 u. ff. 


\section{Versuch 1.}

Zur Verwendung kommt eine Trypsinlösung von $1,0 \%$ in Sodalösung von $0,1 \%$. Die Verdauungsgläschen enthalten je $15 \mathrm{ccm}$ Sodalösung und ausserdem

Gläschen 1: 0 ccm Trypsinlösung,

\begin{tabular}{|c|c|c|}
\hline , & $2: 0,1$ & $\eta$ \\
\hline$"$ & $3: \quad 0,4$ & $"$ \\
\hline & $4: \quad 0,9$ & $"$ \\
\hline " & $5: 1,6$ & $"$ \\
\hline
\end{tabular}

und die nötigen Mengen Sodalösung zur Ergänzung auf gleiche Volumina. In jedes Gläschen kommt $1 \mathrm{~g}$ zerkleinertes Eiweiss. Nach 4 Stunden Verdauung bei $40^{\circ} \mathrm{C}$. findet sich Eiweiss in den 5 Gläschen:

In Gläschen 1 ( 0 Trypsin) 1,74 g Eiweiss also verdaut 0,0 g oder bzw. 0,0 g Eiweiss,

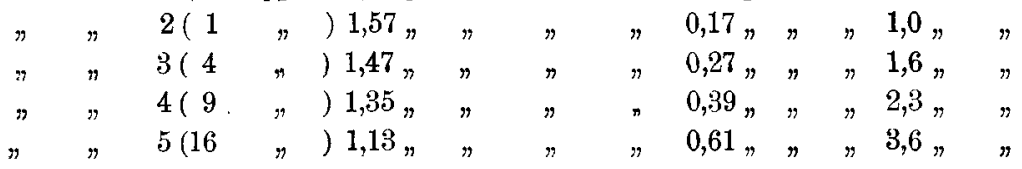

Das Eiweiss quillt in der schwachen Sodalösung ziemlich stark auf, so dass sein Gewicht von $1 \mathrm{~g}$ bis $1,74 \mathrm{~g}$ zunimmt. Dieses Gewicht wurde als Ausgangsgewicht genommen. Die Trypsinmengen verhielten sich in vorstehendem Versuche wie $1: 4: 9: 16$, die verdauten Eiweissmengen wie $1: 1,6: 2,3: 3,6$, ergaben also kleinere Mengen als das Gesetz von Schütz $(1: 2: 3: 4)$ sie verlangte. Lässt man die Verdauung kürzere Zeit dauern, so bleiben die stärkeren Konzentrationen nicht in dem Maasse zurück, sie gehen sogar gelegentlich über die Quadratwurzel hinaus. Nach dreistündiger Verdauung wurden z. B. in gleichartigen Versuchen verdaut die verbältnismässigen Eiweissmengen 1,0, 2,4, 3,0, 4,7 oder ähnliche Mengen.

\section{Versuch 2.}

Ein ganz ähnlicher Versuch mit einer Pepsinlösung in Salzsäure von 0,2\% ergab nach $4^{1 / 2}$ stündiger Verdauung:

In Gläschen 1 ( 0 Pepsin) $1,75 \mathrm{~g}$ Eiweiss also verdaut 0,0 g oder bzw. $0,0 \mathrm{~g}$ Eiweiss,

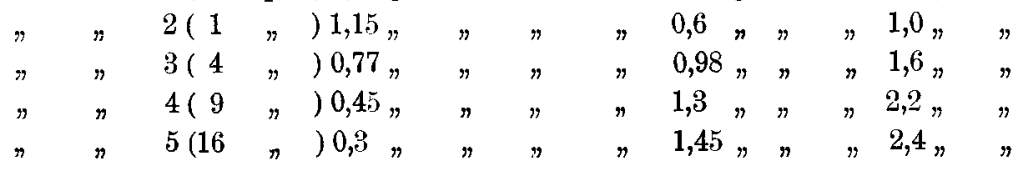

Die verdauten Eiweissmengen bleiben auch hier und zwar noch mehr hinter den Quadratwurzeln der Pepsinkonzentrationen zurück als bei dem Trypsin. Diese Tatsache gilt allgemein bei derartigen Versuchen. Mit dem Trypsin kommt man an das Gesetz von 
S ch ütz viel näher heran als bei ähnlichen Versuchen mit dem Pepsin, eine wichtige Tatsache, die später ihre Erklärung finden wird.

Dass bei allen diesen Gesetzlichkeiten auch die Dauer der Versuche von der allergrössten Wichtigkeit ist, braucht wohl kaum erwähnt zu werden. Von Rechts wegen dürfte man hier überhaupt nur von allgemeinen Gesetzen sprechen, so lange die Oberflächen der zu lösenden Eiweissmengen nahezu gleich sind. Je kleiner sie werden, um so langsamer muss - worauf namentlich Grützner in seiner Arbeit (s. S. 338) hingewiesen hat - auch die Verdaung vor sich gehen. Daher zum Teil das bedeutende Zurückbleiben der höheren Konzentrationen. Es hat aber, wie wir später sehen werden, auch noch andere Gründe.

Vermittelst keiner anderen Methode ist es so leicht, sich von dem Gang der Verdauung eine unmittelbare Anschauung zu verschaffen wie vermittelst der kolorimetrischen. Diese Methode ist es auch (worauf mich Herr Prof. v. Grützner besonders aufmerksam gemacht hat), welche zeigt, dass die Schütz-Borissow'sche Regel nur ein Durchgangsstadium bezeichnet, wenn es sich um die Verdauung von festem Eiweiss handelt. Wie aus allen Versuchen vermittelst der kolorimetrischen Methode hervorgeht, sind die verdauten Eiweissmengen im Anfang der Verdauung etwas grösser als die Quadratwurzeln der Pepsinkonzentrationen; dann werden sie etwa den Quadratwurzeln gleich, und später werden sie immer kleiner als die Quadratwurzeln. Weil diese Verbältnisse für alle sogenannten Fermentgesetze von grosser Wichtigkeit sind, seien sie durch einige Beispiele erläutert. Ich verweise auf den Versuch in Ko rn's Arbeit S. 39, sowie auf meine Versuche 1-3 auf S. 347 u. 348. In denselben habe ich neben die Zahlen meiner Farbenskala, die jenen Zanlen entsprechenden verdauten Eiweissmengen, die erste immer auf 1 berechnet, daneben gesetzt. Man sieht z. B. in Versuch 1, wie die verdauten Fibrinmengen im Anfang durchweg grösser sind als die Quadratwurzeln der Konzentrationen 1,0, 3,6, $5,0,5,8$ statt $1,2,3,4$, wie sie dann um $11^{\text {h }} 5^{\prime}$ in das SchützBorissow'sche Gesetz einmünden, indem sie sich wie 1, 2,1, 3,0, 4,0 verhalten, später aber bedeutend hinter den Quadratwurzeln zurückbleiben; denn sie betragen 1,0, 1,5, 2,25, 3,0. Ganz dasselbe findet statt in den Versuchen 2 und 3 , wie die in Klammern befindlichen arabischen Zahlen zeigen, welche neben den römischen der Farbenskala stehen. 
Unzweifelhaft ist dieser Gang der Verdauung, wie er hier zutage tritt, wesentlich bedingt durch die fortschreitende Verringerung der Oberfläche der Eiweisskörper, welche hier verdaut werden. Wie ist es nun aber, wenn die Oberflächen der Eiweisskörper gleich gross bleiben, wie in den Mett'schen Versuchen?. Auch da tritt mehr oder weniger deutlich das Schutz-Borissow'sche Gesetz in Erscheinung. Es muss also hier noch eine andere, die Verdauung bemmende bzw. verlangsamende Kraft wirksam werden und das sind; wie Grützner für das Pepsin gezeigt hat, die sich bildenden Peptone und wie man noch weiterhin nachweisen kann, der offenbar geringere Pepsingehalt der in den Enden der Glasröhrchen befindlichen Flüssigkeitsmengen. Denn da die Röhrchen ganz ruhig bei derselben Temperatur in ihren Verdauungsgefässen liegen, so ist es klar, dass wesentlich nur durch Diffusion, also sehr langsam, die äussere Flüssigkeit in die Röhrehen eintreten kann. In diesen aber befindet sich ausser dem festen Eiweiss gelöstes Eiweiss + Pepsinlösung, welche von aussen herantritt. Daher kommt es auch, dass die Verdauung um so langsamer fortschreitet, je tiefer hinein sie in die Gläschen eindringt; denn um so mehr wird die Pepsinlösung durch die Peptonlösung verdünnt, und daher kommt es weiter, wie ebenfalls Grützner gezeigt hat, dass in einem senkrecht (oder nahezu senkrecht) stehenden Mett'schen Röhrchen das obere Liweiss viel langsamer verdaut wird als das untere ${ }^{1}$ ). Nur wenn die verdauten Massen bei weiteren Röhren leicht abfliessen und zu Boden sinken können, und wenn weiter die Pepsinlösungen in ausreichender Menge vorhanden sind, dann zeigt sich das Grützner'sche Gesetz, dass die Längen der verdauten Eiweisszylinder (also die

1) Es ist leicht zu zeigen, dass man ähnliche Gesetzlichkeiten auch bei Auflösung von Kristallen in Wasser nachweisen kann: Wenn man sich z. B. einen Kupfersulfatkristall rund feilt, so dass ein etwa zwei Zentimeter langer 4-5 mm dicker Zylinder entsteht, den man an seiner Mantelfläche mit Lack überzieht und ausserdem in ein lackiertes Papier einwickelt, so kann man folgendes beobachten, sobald er senkrecht in ein mit Wasser gefülltes Becherglas aufgehängt wird, und zwar nahe der Oberfläche des Wassers. Von einem solchen Kupfersulfatzylinder war z. B. nach 25 Minuten oben gelöst $0,5 \mathrm{~mm}$, unten $7 \mathrm{~mm}$, von einem anderen

nach 10 Minuten oben $0,0 \mathrm{~mm}$, unten $2 \mathrm{~mm}$

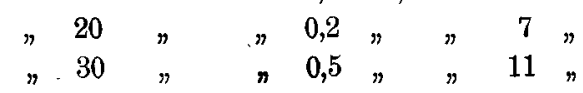

Oben befand sich eben eine mehr oder weniger gesättigte Kupfersulfatlösung, unten eine sehr schwache.! 
eiweissfreien Enden der Mett'schen weiten Röhren) sich zu, den Pepsinkonzentrationen verhalten wie die dritten Wurzeln aus ihren Quadraten oder anders ausgedrückt, dass die in der Zeiteinheit gelösten Eiweissmengen direkt proportional sind den wirksamen Pepsinmengen.

Es fragt sich jetzt weiter, wie liegen diese Vorgänge bei dem Trypsin gegenüber dem Pepsin? Nun in zwei sehr wichtigen Punkten weicht die Wirkung des Trypsins von der des Pepsins ab. Das Pepsin verbraucht sich, wie Grützner ${ }^{1}$ ) gezeigt hat, bei der Verdauung ausserordentlich wenig, das Trypsin aber in hohem Maasse, wie wohl Heidenhain ${ }^{2}$ ) zuerst nachgewiesen und Vernon bestätigt hat. Andererseits aber hemmen die gebildeten Peptone die Pepsinverdauung sehr bedeutend, die Trypsinverdauung aber gar nicht, wovon ich mich ebenfalls vermittelst der kolorimetrischen Methode leicht überzeugen konnte. Jedenfalls schiebt sich also auch bei der Trypsinverdauung eine hemmende Kraft ein, welche namentlich unter gewissen Bedingungen zu einem dem Schütz-Borissow'schen Gesetze ähnlichen führen kann. Denn wenn auch die Oberflächen der zur Verdauung bestimmten Eiweissmassen nahezu gleich bleiben, wie in den Mett'schen Versuchen, soll doch nach Samojloff und Walther ebenfalls das Quadratwurzelgesetz für das Trypsin gelten.

Es interessierte mich, diese Angaben zu prüfen. Die Versuche mit Eiweiss fielen unbefriedigend aus, weil die Verdauung gar zu langsam vorwärts schritt. Man konnte selbst nach mehreren Stunden kaum eine Verdauung nachweisen. Ich versuchte deshalb die Methode von Fermi, welche mir vortreffliche Ergebnisse lieferte. Die Kapillarröhrchen wurden anstatt mit Eiweiss mit flüssigem (nach Fermi's Vorschriften bereitetem, aber thymolfreiem) Leim gefültt und abgekühlt. Den Leim färbte ich vorher mit ein wenig Methylviolett, um die Grenzen des verdauten Leims genauer ablesen zu können, ging aber dann, weil das Methylviolett sich in Wasser löst und die scharfen Grenzen zwischen verdauter Flüssigkeit und Leim verwischt, auch die Verdauung immer etwas hemmte, zur Färbung des Leims mit Spritblau über. Da sieht man die Grenzen zwisehen Leim und Flüssigkeit haarscharf. Auch vollzog sich die Verdauung schneller.

1) P. Grützner, Neue Untersuchungen usw. S. $9 \mathrm{ff}$.

2) R. Heidenhain, Pflüger's Arch. Bd. 10, in der oben genannten Arbeit S. 579. 


\section{Folgende Versuche dienen als Beispiele:}

Versuch 1 (8. Juni, Nr. 25).

5 mit blauem Leim gefüllte Kapillarröhrchen, welche 2 Stunden in $0,1 \%$ iger Sodalösung gelegen hatten, wurden in Gläschen gelegt, die je $16 \mathrm{ccm}$ Flüssigkeit enthielten, und zwar:

Gläschen $10,0 \mathrm{ccm}$ Trypsinlösung $+16,0 \mathrm{ccm}$ Sodalösung von $0,1 \%$,

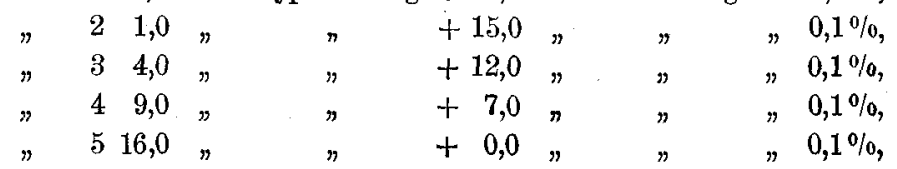

Das Trypsin $1 \%$ war ebenfalls in Sodalösung von $0,1 \%$ gelöst. Die Kapillarröhren lagen auf dem Boden der Gläschen; die Verdauung dauerte 20 Stunden bei einer Temperatur von $24^{\circ}$ C. Es ergab sich folgendes Resultat:

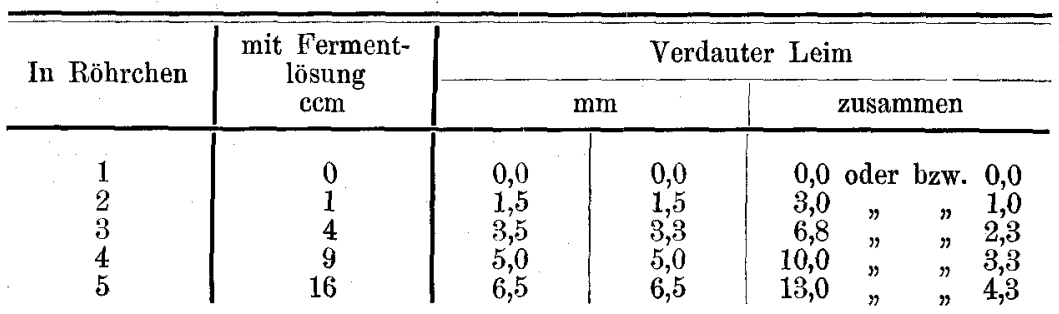

Versuch 2 (7. Juni, Nr. 26).

Eine schwächere Trypsinlösung $(0,5 \%$ Ferment in Sodalösung von $0,1 \%)$ gelangt unter ganz denselben Bedingungen zur Verwendung. Die Verdauung dauert 18 Stunden.

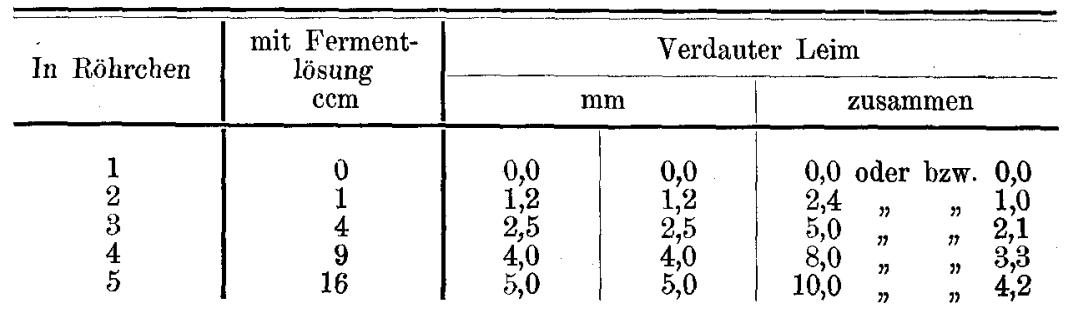

Versuch 3 (7. Juni, Nr. 27).

Eine $1 \%$ ige Trypsinlösung in den gleichen Konzentrationen wie oben verdaute in 18 Stunden bei $25^{\circ} \mathrm{C}$.:

\begin{tabular}{|c|c|c|c|c|}
\hline \multirow{2}{*}{ In Röhrchen } & \multirow{2}{*}{$\begin{array}{l}\text { mit Ferment- } \\
\text { lösung } \\
\text { ccm }\end{array}$} & \multicolumn{3}{|c|}{ Verdauter Leim } \\
\hline & & \multicolumn{2}{|c|}{$\mathrm{mm}$} & zusammen \\
\hline $\begin{array}{l}1 \\
2 \\
3 \\
4 \\
5\end{array}$ & $\begin{array}{r}0 \\
1 \\
4 \\
9 \\
16\end{array}$ & $\begin{array}{l}0,0 \\
1,0 \\
2,3 \\
4,0 \\
5,0\end{array}$ & $\begin{array}{l}0,0 \\
1,3 \\
2,5 \\
4,0 \\
5,0\end{array}$ & $\begin{array}{rcc}0,0 & \text { oder } & 0,0 \\
2,3 & & 1,0 \\
4,8 & " & 2,1 \\
8,0 & " & 3,5 \\
10,0 & \prime & 4,3 \\
* & & \end{array}$ \\
\hline
\end{tabular}


Aus diesen Versuchen sieht man zunächst, wie diese Methode, eine entsprechende Abänderung der M ett'schen, gut für quantitative Trypsinbestimmungen verwertbar ist. Das Quadratwurzelgesetz gilt nahezu; besondere Beachtung aber verdient, dass die verdauten Leimmengen, namentlich die grösseren, nicht wie es bei den Pepsinversuchen für das Eiweiss durchweg gilt, zurückbleiben (s. S. 342 die Zahlen bei Samojloff und Korn), sondern dass sie grösser sind als die Quadratwurzeln der Fermentkonzentrationen. Die Art und Stärke der Hemmung, welche hier in Betracht kommt, ist also eine ganz andere, als bei der Pepsinverdauung. Im grossen und ganzen aber stimmen meine Ergebnisse mit den Angaben von Samojloff und Walther überein.

Anders aber wird die Sache sofort, wenn man, wie das Grützner getan hat, dafür sorgt, dass alle Hemmungen bei der Verdauung so viel wie iinöglich beseitigt werden. Das geschieht am einfachsten, wenn man die Mett'schen Röhrchen senkrecht hängt und zwar in die oberen Scbichten von einer grösseren Menge Verdau ungsflüssigkeit oder wenn überhaupt nur sehr wenig verdaut wird. Dann kommt doch stets, wenn die Röhrchen nicht gar zu eng sind, die ursprüngliche Verdauungsflüssigkeit in unmittelbare Berührung mit dem zu verdauenden Leim. Ich habe ziemlich viel Versuche nach dieser Richtung hin angestellt, und alle mit den gleichen Ergebnissen, nämlich denjenigen, welche das Grützner'sche Gesetz fordert: die verdauten Leimmengen waren ziemlich genau proportional den dritten Wurzeln aus den Quadraten der Konzentrationen. Einige Versuche mögen als Belege dienen.

Versuch 1 (13. Juni, Nr. 32).

Die mit Spritblau-Gelatine gefüllten Röhrchen werden an einem Finde mit einem dünnen Seidenfaden an Holzstäbchen gehängt, welche quer über den mit Verdauungsflüssigkeit gefüllten Bechergläschen liegen, so dass sie möglichst senkrecht herabhängen. Die Verdauung geschieht bei $19^{\circ} \mathrm{C}$. Die Gläschen stehen, wie auch bei allen früheren Versuchen in einem eigens dazu hergerichteten Wasserbad. Der Versuch dauert 25 Stunden.

\begin{tabular}{|c|c|c|c|c|c|}
\hline \multirow{2}{*}{ Glas } & \multirow{2}{*}{$\begin{array}{l}\text { Enthält } \\
\text { Trypsin- } \\
\text { lösung 1\% } \\
\text { ccm }\end{array}$} & \multirow{2}{*}{$\begin{array}{c}\text { Enthält } \\
\text { Sodalösung } \\
0,1 \% \\
\text { cem }\end{array}$} & \multicolumn{2}{|c|}{ Verdaute Mengen Leim } & \multirow{2}{*}{$\left(t=\underset{3}{\sqrt[3]{t^{2}}} \underset{\text { mengen }}{\operatorname{Tryp}}\right.$} \\
\hline & & & $\begin{array}{l}\text { oben } \\
\text { mm }\end{array}$ & $\begin{array}{c}\text { unten } \\
\text { mm }\end{array}$ & \\
\hline $\begin{array}{l}1 \\
2 \\
3 \\
4 \\
5\end{array}$ & $\begin{array}{r}0 \\
1 \\
4 \\
9 \\
16\end{array}$ & $\begin{array}{l}75 \\
74 \\
71 \\
66 \\
59\end{array}$ & $\begin{array}{c}0 \\
0,4(1,0) \\
1,0(2,5) \\
1,8(4,5) \\
2,5(6,2)\end{array}$ & $\begin{array}{ccc}0,0 & \text { oder } & 0,0 \\
0,9 & \Rightarrow & 1,0 \\
2,5 & \Rightarrow & 2.8 \\
3,6 & \Rightarrow & 4,0 \\
5,3 & \# & 5,9\end{array}$ & $\begin{array}{l}0,0 \\
1,0 \\
2,5 \\
4,33 \\
6,35\end{array}$ \\
\hline
\end{tabular}


Versuch 2 (13. Juni, Nr. 33).

Ein gleicher Versuch unter denselben Bedingungen an demselben Tage angestellt, ergibt:

\begin{tabular}{c|c|c|c|c}
\hline \hline \multirow{2}{*}{$\begin{array}{c}\text { Ferment- } \\
\text { mengen }\end{array}$} & \multicolumn{2}{|c|}{$\begin{array}{c}\text { Verdaute Mengen Leim } \\
\text { oben } \\
\text { mm }\end{array}$} & $\begin{array}{c}\text { unten } \\
\text { mm }\end{array}$ & $\sqrt[3]{t^{2}}$ \\
\hline 0 & 0,0 & 0,0 oder 0,0 & 0,0 \\
1 & $0,5(1,0)$ & 0,9 & 1,0 & 1,0 \\
4 & $1,0(2,0)$ & 2,2 & 2,4 & 2,5 \\
9 & $1,8(3,6)$ & 5,5 & $3,5,4$ & 4,33 \\
16 & $2,5(5,0)$ & 5,5 & 6,1 & 6,35
\end{tabular}

Wie man also sieht, folgen namentlich die unten abverdauten Leimmengen dem Grützner'schen Gesetz. Bei den oberen pflegen (Versuch 2) die grösseren Mengen ein wenig zurückzubleiben. Dieses Zurückbleiben wird aber ausserordentlich stark, sobald die Verdauung in etwas höherer Temperatur geschieht, was wohl auf die stärkere Zerstörung des T'rypsins zurückgeführt werden dürfte. Folgende Versuche zeigen dies.

Versuch 3 (15. Juni, Nr. 34).

Anordnungen wie in Versuch 1 und 2. Daner des Versuches 23 Stunden. T'emperatur $25{ }^{\circ} \mathrm{C}$. Dieselben Fermentmengen in denselben Flüssigkeitsmengen.

\begin{tabular}{|c|c|c|}
\hline $\begin{array}{l}\text { Ferment- } \\
\text { mengen }\end{array}$ & \begin{tabular}{c|c} 
Verdante & Mengen Leim \\
oben & unten \\
$\mathrm{mm}$ & $\mathrm{mm}$
\end{tabular} & $\sqrt[3]{t^{23}}$ \\
\hline 0 & $\begin{array}{c:c}0,0 & 0,0 \\
\text { etwas heraus gequollen }\end{array}$ & 0,0 \\
\hline $\begin{array}{r}1 \\
4 \\
9 \\
16\end{array}$ & \begin{tabular}{l|ccc}
1,0 & 1,1 & oder & 1,0 \\
1,5 & 2,9 & $n$ & 2,6 \\
2,1 & 4,3 & $n$ & 8,9 \\
2,5 & 6,3 & $n$ & 5,7
\end{tabular} & $\begin{array}{l}1,0 \\
2,5 \\
4,3 \\
6,35\end{array}$ \\
\hline
\end{tabular}

Versuch 4 (15. Juni, Nr. 35).

Derselbe Versuch wiederholt. Dauer 23 Stunden. Temperatur $25^{\circ}$ (., einige Zeit etwas niedriger.

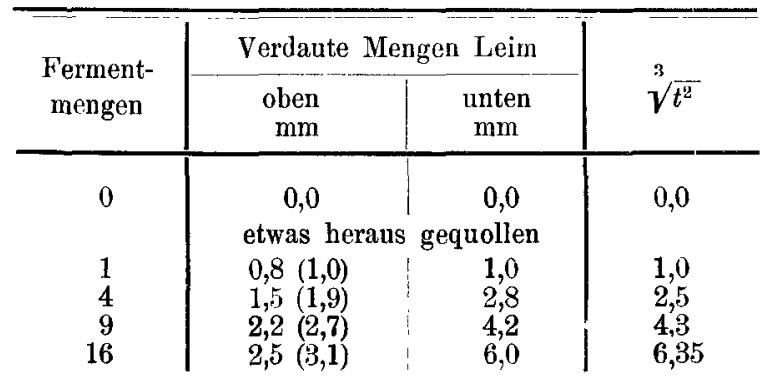


Es wird nicht nötig sein, noch mehr Versuche derart mitzuteilen. Sie alle zeigen, abgesehen von der viel besseren Verdauung unten als oben, dieselben Gesetzlichkeiten. Und es stimmen die gefundenen Zahlen, auch die nicht mitgeteilten, recht gut mit den berechneten, namentlich wenn man bedenkt, dass die Messung der verdauten Leimsäulchen nicht aufs Haar genau geschehen kann und Fehlerquellen hier wie bei jeder anderen Methode unvermeidlich sind.

Erwähnt sei noch, dass, wenn man dieselben Versuche mit Pepsinlösungen und Mett'schen Eiweissröhrchen macht, sie anders ausfallen, d. h. dass die von den stärkeren Pepsinlösungen verdauten Eiweissmengen bedeutend zurückbleiben, wie folgender Versuch zeigt.

\section{Versuch.}

Pepsinextrakt in Salzsäure von $0,2 \%$ je $25 \mathrm{ccm}$ in fünf Gläschen, in denen die Mett'schen Röhrchen oben senkrecht hängen. Dauer des Versuches 22 Stunden, Temperatur $37^{\circ} \mathrm{C}$.

\begin{tabular}{|c|c|c|c|}
\hline \multirow{2}{*}{$\begin{array}{l}\text { Ferment- } \\
\text { mengen }\end{array}$} & \multicolumn{2}{|c|}{ Eiweiss verdaut } & \multirow{2}{*}{$\begin{array}{c}{\sqrt[3]{P^{2}}}^{\sqrt{2}} \\
=\text { mengen }\end{array}$} \\
\hline & $\begin{array}{c}\text { oben } \\
\text { mm }\end{array}$ & $\begin{array}{l}\text { unten } \\
\text { mm }\end{array}$ & \\
\hline $\begin{array}{r}0 \\
1 \\
4 \\
9 \\
16\end{array}$ & $\begin{array}{ccc}0,0 & \text { oder } & 0,0 \\
1,5 & \# & 1,0 \\
2,4 & \# & 1,6 \\
2,6 & \# & 1,7 \\
3,0 & \# & 2,0\end{array}$ & $\begin{array}{ccc}0,0 & \text { oder } & 0,0 \\
1,5 & \# & 1,0 \\
2,4 & \Rightarrow & 1,6 \\
4,0 & \# & 2,7 \\
4,8 & \# & 3,2\end{array}$ & $\begin{array}{l}0,0 \\
1,0 \\
2,5 \\
4,33 \\
6,35\end{array}$ \\
\hline
\end{tabular}

Lehrreich ist das Zurückbleiben der Verdauung in den Röhrchen oben, je weiter die Verdauung bei späteren Konzentrationen fortschreitet. Hier sind die Hemmungen offenbar in den engen Kapillarröhrchen am bedeutendsten. Will man bei der Pepsinverdauung die reine Abhängigkeit der verdauten Eiweissmengen von den tätigen Fermentmengen studieren, so muss man, wie das Grützner getan, nicht kapillare Röhrehen, sondern weite Röhren verwenden.

Aus allen diesen Versuchen geht also hervor, dass für das Trypsin ganz dasselbe Gesetz gilt wie für das Pepsin, wenn man berücksichtigt, wie viel Fermentmoleküle überhaupt auf die Oberfläche des zu verdauenden Eiweisses wirken können. Dabei muss natürlich diese Oberfläche konstant erhalten, und ferner müssen alle Widerstände, die sich der Verdauung entgegenstellen, wie die Vermischung der Verdauungsflüssigkeit mit Peptonen, Zerstörung von Ferment, die Änderung der Konzentration der Fermentlösung u. a., möglichst vermieden werden. Ein Fermentmolekül leistet daun dieselbe Arbeit wie jedes andere, $\mathrm{n}$ wirksame Fermentmoleküle n-mal 
so viel wie eins. Wirksam aber können nur diejenigen Fermentmoleküle sein, welche mit der Oberfläche des Eiweisses in unmittelbare Berührung kommen. Daher verdaut eine Fermentlösung, in welcher n-mal so viel Fermentmoleküle sind als in einer anderen unter sonst gleichen Bedingungen niemals n-mal so viel festes Eiweiss, sondern nur 3 $\sqrt{n^{2}}$ mal so viel.

Ganz das gleiche muss natürlich auch gelten, wenn man nicht, was - wie oben schon auseinandergesetzt - im allgemeinen viel zweckmässiger ist, die Verdauungszeiten gleich lässt und nach den in diesen Zeiten verdauten Eiweissmengen fragt, sondern die Frage aufwirt, in nerhalb welcher \%eit verschiedene Fermentmengen dieselbe Arbeit verrichtet haben, also z. B. gleiche Mengen von Eiweiss verdaut haben. Selbstverständlich müsste man auch bei derartigen Versuchen, wenn man irgend ein Gesetz herausfinden wollte, für gleiche Oberfläche des zu verdauenden Eiweisses und für Vermeidung aller Hemmungen während der Verdauung sorgen. Derartige Versuche hatte ich noch nicht Gelegenheit anzustellen. Sie würden aller Wahrscheinlichkeit dieselben Gesetzlichkeiten ergeben.

Berücksichtigt man aher diese Vorsichtsmaassregeln nicht, so ergeben sich, wie längst bekannt ist, ganz andere Beziehungen zwischen Fermentmenge und Geschwindigkeit der Verdauung. Es ist behauptet worden, dass die $n$-fache Menge des Fermentes eine bestimmte Menge von Eiweiss in der n-mal so kurzen Zeit wie die einfache löst. Daran ist aher gar nicht zu denken, sondern sie braucht viel länger dazu. $B \mathrm{rück}^{1}$ ) verwendete z. B. Pepsinlösungen, welche sich wie $1: 2: 4: 8: 10: 32$ verhielten; die Verdaungsgeschwindigkeiten (das sind die umgekehrten Zeiten) zeigten aber keineswegs dieselben verhältnismässigen Grössen, sondern die achtfache Pepsinmenge verdaute etwa nur $4,7 \mathrm{mal}$ so schnell als die einfache. In einem Versuche von $\mathrm{May}$ e ${ }^{2}$ ) dagegen verdauten die $16,8,4,2$ fachen Pepsinmengen bezüglich nur $2,8,2,3,2,0,1,7 \mathrm{mal}$ so schnell als die einfachen.

Auch ich stellte einige dahingehende Versuche mit 'Trypsin und Pepsin an und will zwei von ihnen mitteilen.

1) Siehe P. v. Grützner, Versuche und Betrachtungen usw. Arch. di Fisiol. vol. 7 p. 223.1909.

2) Siehe P. v. Grützner, Versuche und Betrachtungen usw. Arch. di Fisiol. vol. 7 , p. 2283.1909. 


\section{Versuch 1. (27. Mai, Nr. 19.)}

Gleiche Mengen Spritblaufibrin werden mit je $15 \mathrm{ccm}$ von verschieden starken Trypsinlösungen in Brutwärme verdaut. In den Gläschen befinden sich $0,4,0,9$ und 1,6 ccm Trypsinlösung ( $1 \mathrm{~g}$ Trypsin zu $100 \mathrm{ccm}$ Sodalösung von $0,1 \%$ mit den entsprechenden Mengen gleicher Sodalösung versetzt. Das Fibrin war aufgelöst nach bezüglich 6 Stunden 50 Minuten,

$\begin{array}{lllll}4 & n & 40 & & \\ 3 & n & 25 & n\end{array}$

Die Verdaungsgeschwindigkeiten verhielten sich sonach wie $1,0: 1,46: 2,0$,

"Trypsinmengen $\quad " \quad " \quad 1,0: 2,25: 4,0$.

Versuch 9. (28. Mai, Nr. 20.)

Fin ganz ähnlicher Versuch mit Pepsin, in welchem sich die Mengen des Pepsins in je $25 \mathrm{ccm}$ Salzsäure wie 1:4:9:16 verhielten und gleiche Mengen Karminfibrin bei Zimmertemperatur verdaut wurden, zeigte folgende Ergebnisse. Die Zeiten, in denen alles Fibrin verdaut war, betrugen bezüglich 2 Stunden 58 Minuten,

1 Stunde 8

$38 \quad "$

Die Verdauungsgeschwindigkeiten also bezüglich $1,0: 2,6: 4,7: 7,1$.

"Pepsinmengen, wie oben mitgeteilt $\quad 1: 4: 9: 16$.

Auch in diesen Versuchen bestand also keineswegs eine direkte Proportionalität zwischen Fermentmengen und Verdauungsgeschwindigkeiten. Die grösseren Fermentmengen verdauten verhältnismässig viel zu laugsam.

Auch wenn man, was sich mit der kolorimetrischen Methode sehr leicht ausführen lässt, nicht bis zu dem Ende der Verdauung wartet, sondern nur so lange, bis in allen Verdauungsgläschen, welche gleiche Mengen Fibrin, aber versehiedene Mengen Ferment enthalten, gleich viel Fibrin gelöst worden ist, also die Frage beantwortet, innerhalb welcher Zeit in den verschiedenen Gläschen eine bestimmte Farbe erreicht ist, erhält man dieselben Verhältnisse. Die starken Fermentlösungen bleiben ungeheuer zurück. Die Farbe II meiner Skala wurde z. B. in einem Versuch mit den Trypsinmengen 1, 4, 9, 16 erreicht in beziehungsweise $60,40,25$ und 18 Minuten. Den genannten Fermentmengen entsprechen also die Verdauungsgeschwindigkeiten $1,0,1,4,2,2,3,3$.

Nachdem wir also gesehen haben, dass bei den gebräuchlichen Verdauungsversuchen nahezu die Schütz-Borissow'sche Regel, unter bestimmten Versuchsbestimmungen aber, wie Gleicherhaltung der Oberfläche des zu verdauenden Eiweisses und Vermeidung aller 
Hemmungen das Grützner' sche Gesetz recht genau gilt, kommen wir zum Schluss zu der wichtigen Frage, wie sind die Angaben von Volhard und seinen Schülern sowie diejenigen von Gross zu erklären, welche auf das bestimmteste eine direkte Proportionalität zwischen Fermentmenge und Verdauungsgeschwindigkeit behaupten.

Nun, der wesentliche Unterschied in jenen beiden Versuchsarten ist der, dass die letztgenannten Forscher flüssiges Eiweiss, die anderen aber so wie ich in den oben beschriebenen Versuchen festes Eiweiss zur Verdauung verwendeten. Ich machte mich daher daran, die Versuche von Gross zu wiederholen, weil mir die Methode als die einfachste von den genannten gleichartigen ersehien. Und siehe da, auch ich kam zu ganz den gleichen Ergebnissen wie Gross; das in schwacher Sodalösung (von $0,1 \%$ gelöste Kasein wurde um so schneller gelöst, je mehr Trypsin in der Lösung sich fand, und die Lösungsgeschwindigkeiten waren den Fermentmengen direkt proportional. Weder bestand das Schütz-Borissow'sche noch das Grützner'sche Gesetz. Nun, dass das letztere hier nicht gelten konnte, ist klar; denn bei dieser Art von Verdauung gibt es kein zu verdauendes Eiweissstück mit gleichbleibender Oberfläche, sondern gelöstes Fiweiss, dessen Oberfläche wir, wenn auch nicht als unendlich, doch als sehr gross ansehen können. Nahezu an jeder Stelle des Raumes in der Verdauungsflüssigkeit können die ebenfalls durch den ganzen Raum verteilten Fermentmoleküle mit den Fiweissmolekülen in Berührung kommen. Je mehr Fermentmoleküle vorhanden sind, um so mehr Eiweissmoleküle können von ihnen gepackt werden.

Die $\mathrm{n}$ fache Menge der Fermentmoleküle kann $\mathrm{n}$-mal so viel Eiweissmoleküle angreifen und in derselben Zeit also n-mal so viel Eiweiss auflösen oder mit einer bestimmten Eiweissmenge in n-mal so kurzer Zeit fertig werden.

Dass dies alles in der Tat für das Trypsin gilt, haben die genannten Forscher gezeigt. Es ist ein leichtes, sich vermittelst der Methode von Gross von der Richtigkeit dieser Tatsachen zu überzeugen. Folgender Versuch diene als Beispiel.

\section{Versuch 1.}

Es wird Caseinum purissimum nach Hammarsten von Grübler in $0,1 \%$ iger Soda aufgelöst $(1: 1000)$. Je $10 \mathrm{ccm}$ dieser Lösung werden in 5 Gläschen verteilt und mit versshiedenen Mengen einer Trypsinlösung (1 Trypsinum siccum Grübler in 100 derselben Sodalösung) versetzt und zwar mit 0,1, 0,2, 0,4 und $0,8 \mathrm{ccm}$ Trypsinlösung und mit Sodalösung zu je $15 \mathrm{ccm}$ aufgefüllt. Die 
Verdauung geschieht in Körpertemperatur und beginnt um $2^{\text {h }} 57^{\prime}$. Sie war beendet bezüglich nach 101, 50, 25, 12 Minuten ${ }^{1}$ ). Die Verdauungsgeschwindigkeiten verhielten sich also wie $1: 2: 4: 8,4$, also nahezu wie die Fermentmengen. Andere ähnliche Versuche lieferten dieselben Ergebnisse.

Auch den Versuch von Gross, dass die n-fache Kaseinmenge n-mal so viel Zeit gebraucht, um von einer bestimmten Fermentmenge aufgelöst zu werden, als die einfache, konnte ich leicht bestätigen. Er ist auch leicht zu verstehen, da eben, wie man doch annehmen muss, eine gegenseitige Durchdringung der beiden Flüssigkeiten stattfindet und verhältnismässig um so weniger tätige Fermentmoleküle vorhanden sind, je grösser die Zahl der Kaseinmoleküle wird.

Wie himmelweit versehieden derartige Versuche ausfallen, wenn statt des gelösten Eiweisses festes Eiweiss verwendet wird, ist schon lange bekannt. So behauptet Brücke in seinen bekannten "Beiträgen zur Lehre von der Verdauung", dass eine grosse Menge von in Salzsäure gequollenem Fibrin sich nahezu ebenso schnell auflöst wie eine kleine Fiòrinflocke, wenn beide von der gleichen Menge Pepsin verdaut werden. Wenn auch dieser Versuch keine reinen Bedingungen darbietet, so ist doch, wie Grützner ${ }^{2}$ ) gezeigt hat, so viel sicher, dass, wenn man verschieden grosse Fibrinmengen in ausreichend viel Pepsinlösung der gleichen Konzentration legt, in breiten Grenzen die Zeitunterschiede der vollständigen Verdauung ziemlich geringe sind, ja bei stärkeren Pepsinlösungen ganz verschwinden können, wovon auch ich mich überzeugte.

Wie steht es nun aber bei dem Trypsin? Gelten da dieselben Verhältnisse oder andere? Wiederum gaben mir Versuche mit der kolorimetrischen Methode in sehr einfacher Weise ganz bestimmte Antworten. Ein Versuch diene als Beispiel.

Versuch. (11. Juni, Nr. 28.)

In 4 Reagenzgläsern werden verschiedene Mengen von Spritblaufibrin, die sich verhalten wie $1: 2: 3: 4$, mit je $15 \mathrm{~cm}$ derselben Trypsinlösung (1,0 Trypsin zu 100 Sodalösung von $0,1 \%$ ) übergossen und der Verdauung in der Wärme ausgesetzt. Nach einer Viertelstunde zeigen die Gläschen sehr verschiedene Färbungen, nämlich I, II, II-III, III-IV.

1) Da man aus anderen ähnlichen Versuchen ungefähr die Dauer der Verdauung kannte, goss man um diese Zeit eine kleine Menge der Verdauungsflüssigkeit aus dem Gläschen in andere Gläschen und fügte ein paar Tropfen verdünnter Essigsäure hinzu. Fehlte jedweder Niederschlag,. so war für mich die Verdauung beendet.

2) P. v. Grützner, Neue Untersuchungen usw. S. 12 u. ff. 
Wäre der Vorgang der Verdauung so wie in den oben beschriebenen Versuchen mit dem flüssigen Kasein, so müsste man erwarten, dass in gleichen Zeiten etwa gleiche Mengen verdaut würden; denn die n-fache Menge Eiweiss verlangt ja n-mal so viel Zeit zur Verdauung. Wenn das Fibrin in dem ersten Gläschen mit der geringsten Fibrinmenge verdaut ist, dürfte auch in den anderen Gläschen nicht mehr verdaut sein; die Farben in den Gläschen müssten gleich sein. Daran ist aber, wie gesagt, nicht zu denken. Durch den ganzen Versuch hindurch sind von Anfang bis zu Ende die Gläschen mit den grösseren Fibrinmengen dunkler als die mit den geringeren, natürlich wegen der grösseren Oberfläche des zu lösenden Fibrins. Und da in ihnen die Verdauungsgeschwindigkeit viel grösser ist als in den anderen mit weniger Fibrin, so werden sie auch in nahezu derselben Zeit mit ihrem Fibrin fertig. In obigem Versuch dauerte es z. B. 5 Stunden, bis in dem ersten Gläschen, und etwa noch 30 Minuten länger, bis auch in dem letzten die Verdaunng beendet war. Es war natürlich gar keine Rede davon, dass die vierfache Menge Fibrin viermal so viel Zeit zur Verdauung gebraucht hätte wie die einfache.

Fasse ich hiernach die Ergebnisse meiner Arbeit, die ich leider bei der mir zugemessenen kurzen Zeit nur auf das Trypsin ${ }^{1}$ ) ausdehnen konnte, kurz zusammen, so dürften sie folgendermaassen lauten.

1. Dadurch, dass man das zu verdauende Fibrin mit einem passenden Farbstoff, Spritblau bläulich, färbt, ist es möglich, die Lösung von äusserst geringen Fibrinmengen, welche die Flüssigkeit blau färben, nachzuweisen. Diese Methode ist also sehr genau. Da ausserdem die Verdauung um so schneller vor sich geht, je mehr Ferment in Tätigkeit ist, so kann man aus der mehr oder weniger starken Färbung, ganz wie bei der kolorimetrischen Pepsinbestimmung von Grützner, die Schnelligkeit der Verdauung und demgemäss den relativen Fermentgehalt der Lösungen schnell und sicher bestimmen. Diese Methode wird von keiner anderen quantitativen übertroffen.

1) Wie mir soeben Herr Prof. v. Grützner mitteilt, dürften in kurzem ähnliche Untersuchungen von anderen Fermenten aus seinem Institut veröffentlicht werden. 
364 Alex. Palladin: Über eine einfache quant. Trypsinbestimmung etc.

2. Das sogenannte Fermentgesetz des Trypsins lautet genau so wie das von Grützner festgestellte des Pepsins. Handelt es sich nämlich um die Verdauung von festem Eiweiss (oder Leim), und sorgt man dafür, dass möglichst alle Störungen und Hemmungen vermieden werden und stets eine gleichgrosse Fläche Eiweiss dem Ferment dargeboten wird, so sind die verdauten Eiweissmengen proportional der Kubikwurzel aus dem Quadrat der Fermentmengen. Verdaut man auf gewöhnliche Weise, indem man das zerkleinerte Fiweiss einfach in die Verdauungsflüssigkeit bringt oder nach Mett'scher Art Röhrchen damit (oder besser mit buntem Spritblauleim) anfüllt, so gilt ziemlich genau das Schütz-Borissow'sche Quadratwurzelgesetz. Denn in beiden Fällen werden hier der Verdauung Hemmungen oder andere Vorgänge, wie Verkleinerung der Oberfläche des zu verdauenden Eiweisses u. a. entgegengesetzt, welche bewirken, dass in einer bestimmten Zeit weniger verdaut wird, als nach dem Grützner'schen Gesetz verdaut werden sollte, nämlich etwa nur eine den Quadratwurzeln der Fermentkonzentrationen proportionale Menge.

Ist dagegen das zu verdauende Eiweiss gelöst, so zeigt sich, dass bei den von mir angewendeten Versuchsbedingungen eine direkte Proportionalität zwischen den Fermentmengen und den von ihnen gelösten Eiweissmengen besteht. Die n-fache Menge Ferment arbeitet n-mal so schnell wie die einfache.

Hiernach hätten also beide oben genannten Parteien recht, sowohl diejenige, welche behauptet, dass für das Trypsin das Schütz-Borissow'sche, als auch diejenige, welche meint, dass das Volhard'sche Gesetz (denn Volhard hat wohl zuerst das Proportionalitätsgesetz ausgesprochen) gelte. Es kommt eben bloss auf die Methode an, welche man anwendet. Das Wesentliche hierbei aber scheint mir, dass stets ein Fermentmolekül ganz so viel leistet wie jedes andere, also n-Moleküle n-mal so viel wie eines, falls sich seiner Wirkung nicht besondere Hemmungen entgegenstellen und die Fermentmoleküle auch an ihr Opfer (wenn ich so sagen darf), das ist das Eiweiss, herankommen können, wie das alles Grützner zuerst für das Pepsin nachgewiesen hat. 